\title{
SOC Estimation of Non-Linear Lithium-Ion Battery using Modified Coulomb-Counting Method with RUL
}

\author{
Umang Dongre
}

\begin{abstract}
Batteries are preferred source of energy in recent few decades, the development is vast. New development comes with new challenges, the coulomb counting method used to study battery behavior with an accurate measurement mechanism like estimating battery state of charge (SOC), battery's remaining useful life (RUL), working condition and changing tendency.

To accomplish this experiment and algorithm efficiency, we used PIC18F MCU with IoT device to send data to the web server using GPRS, and can be utilized on an Electronic Vehicles (EV) and portable devices in real time to show what's really happening within the battery. The significant challenge of this method is, we might receive accumulative errors associated with initial SOC also the faults of quantities are undefined, hence to get over these restrictions, a part wise linear imprecise used with inconsistent constants to define the integrally non-linear relation amongst the SOC and open circuit voltage (VOC).

In past few decades, Li-ion batteries has developed much consideration in EV applications as well as for mobile phone market due to its many advantages like, quick charging ability, more durable, light weight, good energy density, low rate of self-discharge etc. The SOC is key principle to find, control of Li-ion battery performance which is current area of interest in this publication. This paper will demonstrate how the SOC is controlled, observed with the help of Coulomb-counting algorithm.
\end{abstract}

Index Terms: Lithium-ion battery, Coulomb-counting, State of charge, Part wise linearization, RUL, Hardware implementation.

\section{INTRODUCTION}

For a long time in human history, the rechargeable battery being as a vital part in our day to day life. As a moveable, multipurpose and generally utilized energy storage device. In past few decades battery technologies and materials dramatically improving the charging and discharging performances and enhanced with considerably better life-cycle. Among many varieties of rechargeable batteries are used in manufacture such as Lead-acid and Li-ion, The Li-ion batteries contains extended battery life, more power as well energy as compare to other batteries [1]. It is the most well-known energy-storaging device, which holds up promising potential for the comprehension of the quick advancing brilliant network idea and charged transportation

Revised Manuscript Received on December 30, 2019.

* Correspondence Author

Umang Dongre, Department of Electronics, RTMNU, Nagpur (Maharashtra) India.

(C) The Authors. Published by Blue Eyes Intelligence Engineering and Sciences Publication (BEIESP). This is an open access article under the CC BY-NC-ND license (http://creativecommons.org/licenses/by-nc-nd/4.0/)

systems. Battery innovation is expanding in all respects to deliver cells as fast as possible with greater vitality and density of power to diminish the costs. These most important improvements has been accomplished in Li-ion battery innovation [2], and thus are embraced in different applications with high power and energy, for example, Hybrid electric vehicle (HEV), Telecom towers; uninterruptible power supplies (UPS), portable devices. The battery management systems (BMS) feature is to us e batteries to exceed the cut off estimation of the battery cell packs. Without occurring any damage to the battery some BMS are having another feature too that of condition checking, fault finding, battery state of charge (SOC) as well as state of health (SOH) estimation [21]. If we know state of charge in $\mathrm{EV}$ which gives the data of a car and will know how much further it can travel, and till what degree the battery will get charged. Likewise, exact SOC confirmation is an earnest problem. These issues have been inspecting by researchers. Incorrect estimation may cause over-charging or discharging of a battery [4]. Exactness in SOC estimation provides the required energy accuracy consumption of battery, which helps the device regulator to keep away battery from permanent harms, help battery's internal structure, plus assure optimal battery usage. In literature, several SOC estimation techniques are available [9]: electrochemical [6], book-keeping [7], [8], [9] model based [10], data-oriented [11], etc. As we want to develop a co-operative solution proposing between accuracy, simplicity, its strengths and weaknesses, we use book-keeping approach for this instance.

In book-keeping approach, we will explore the Coulomb-counting algorithm which significantly offers an effective SOC estimation. To increase accuracy and effectiveness using this approach, we first try to overcome its disadvantages using part-wise linear relationship mapping among SOC and Open Circuit Voltage (VOC).

So throughout the experiment, the idea is to apply this algorithm on any simulated hardware platform (for e.g. integrated microcontroller) under considerable computation and ambient temperature to extract plausible data (such as accurately estimated SOC) that would help us to understand Li-ion batteries more and its lifespan and improve our vision towards better science.

The rest of this paper prearranged the following way. Section 2 is committed to the estimation of the SOC. The proposed actual algorithm based on Coulomb-counting will be presented in Section 3 and will illustrate in detail step-by-step an initial SOC-VOC relationship. In Section 4, the purpose is to clarify the implementation of the hardware and to send the data to the web server, and lastly, the paper will conclude the research. 


\section{SOC ESTIMATION}

State of Charge is usually categorized as the percentage of the charge sum present in a battery cell at a specific time to its nominal capacity, for instance the amount of charge that can be kept in the cell when it is completely charged. In fact, this is true just toward the start of the battery life, as the actual cell capacity gradually diminishes with time. The estimation precision of SOC doesn't just provide data about the enduring worthwhile capacity yet additionally show the charge and discharge strategies, which impressively influence the battery [5]. Subsequently, a Lithium-ion battery could have unique capacities because of aging, ambient temperature and self-discharge impacts [9]. The mathematical methods for estimation of SOC are diverse and they are well-ordered by their approach. The arrangement of these SOC estimation approaches is divergent in the several literary works and displayed in Table 1. The direct measurement method gives better precision because an electrolytic physical property partakes in the response during charge and discharge. It is possible to define the linear relationship between acid density change and SOC, However, they are difficult to perform since access to the chemical structure in online inspection systems is not obvious [6]. There is roughly a straight relationship among the SOC and VOC [5]. In reality, it cannot be the identical for full batteries. The conventional VOC-SOC then compares between batteries; there is a topic where the relationship must be estimated precisely to the SOC [5]. The model based methods use several comparable circuit models and algorithms are broadly utilized in EV to assessment the battery SOC. To optimize a battery's polarization qualities, an indistinguishable model is needed to caricaturize the execution of the battery. In this way numerous models types were proposed in writing [13]. For a versatile algorithm for the most part dependent on state observers we need this methods, for example, Coulomb counting and EMF Combination [5], [16] Per-unit system, EKF combination, Terminal Voltage and Internal Resistance [17]. Accordingly, their precision relies upon the proficiency of the battery model and the quality of its portrayed parameters. The data-oriented method gives battery exhibitions with incredible exactness and can alter its structure to foresee errors. So essentially it subject to artificial intelligence algorithm likes neural network [18] and fuzzy logic [19], for a comprehensive range of batteries SOC estimation assumes as a secret features data requirement about internal behaviors. These approaches need a countless of training data. In this way, we need efficient and expensive processing, and the reliability of the learning data [9].

The method of coulomb-counting measures the remaining capacity simply by measuring the transferred charge during battery charging and discharge. This approach, which involves long watching and recalling, was acknowledged as irrational but crucial in checking the reliability of estimated results from dissimilar methods for constant SOC estimation. On the other hand, the ongoing utilizations of battery power in several versatile gadgets and EV [1], [2] basically comprise of calculable equipment and an enormous size of memory, empowering the affirmation of the coulomb counting method [7].

The exactness of the coulomb tallying method practically resorts to a reliable estimate of the battery current and exact estimation of the original SOC [28]. With a pre-known capacity that can be stored in the memory or calculated by the working conditions from the start, the SOC of a battery can be measured by integrating the charging and discharging currents over the function period. [28]. Regardless, the releasable charge is in every case not exactly the put away charge in a charging and discharging cycle. As it were, there are some losses for the duration of charging and discharging. These losses likewise with self-discharging reason collecting errors [28]. For more precise SOC estimation, these components ought to be considered. Moreover, estimation instruments ought to be re-adjusted and the decaying of the releasable capacity ought to be considered for more and more accurate estimation [7]. These non-model-based approaches may collect errors brought about by measurements, conceivable embedded noise and an inaccurate initial SOC [9].

In this paper the determination is to suggest a precise monitoring solution for HEV, Telecom tower, multimedia applications, which may give an exact SOC sign and give battery health order information to achieve the best use of the Li-Ion battery throughout its lifetime. The entire project consists mainly three stages. In the first we access the initial SOC which isn't assured. In the second step coulomb-counting calculations for SOC estimation. Intend to propose a battery model and a versatile calculation to improve the precision.

Supposing that the initial value $S O C_{0}$ is identified rest of the capacity available battery that can be considered by measuring the rate of current flow and integrating it over the time interval $d t$ during charging and discharging [9].

$$
S_{\text {OC }}(t)=S O C_{0}+\frac{1}{C_{\text {nот }}} \int_{t_{0}}^{t} I_{b} d t * 100
$$

$S_{O C}$ : State of Charge

$I_{b} \quad$ : Battery current value

$c_{\text {nom }}$ : Battery nominal capacity

We offer a modified Coulomb counting algorithm of SOC estimation for Lithium-ion batteries in a hardware board which is dependent on microcontroller and it tends to be utilized as real instrument for EV, Telecom tower and media applications. Telecom tower and multimedia applications. Therefore the precision of this algorithm is ensured with the assistance of relation between SOC and VOC a part-wise model classifies the initial SOC, to get over increasing errors because of an ultimate inexactness of sensors and a temperature monitoring by adaptive constant needs a monthly recalibration. 


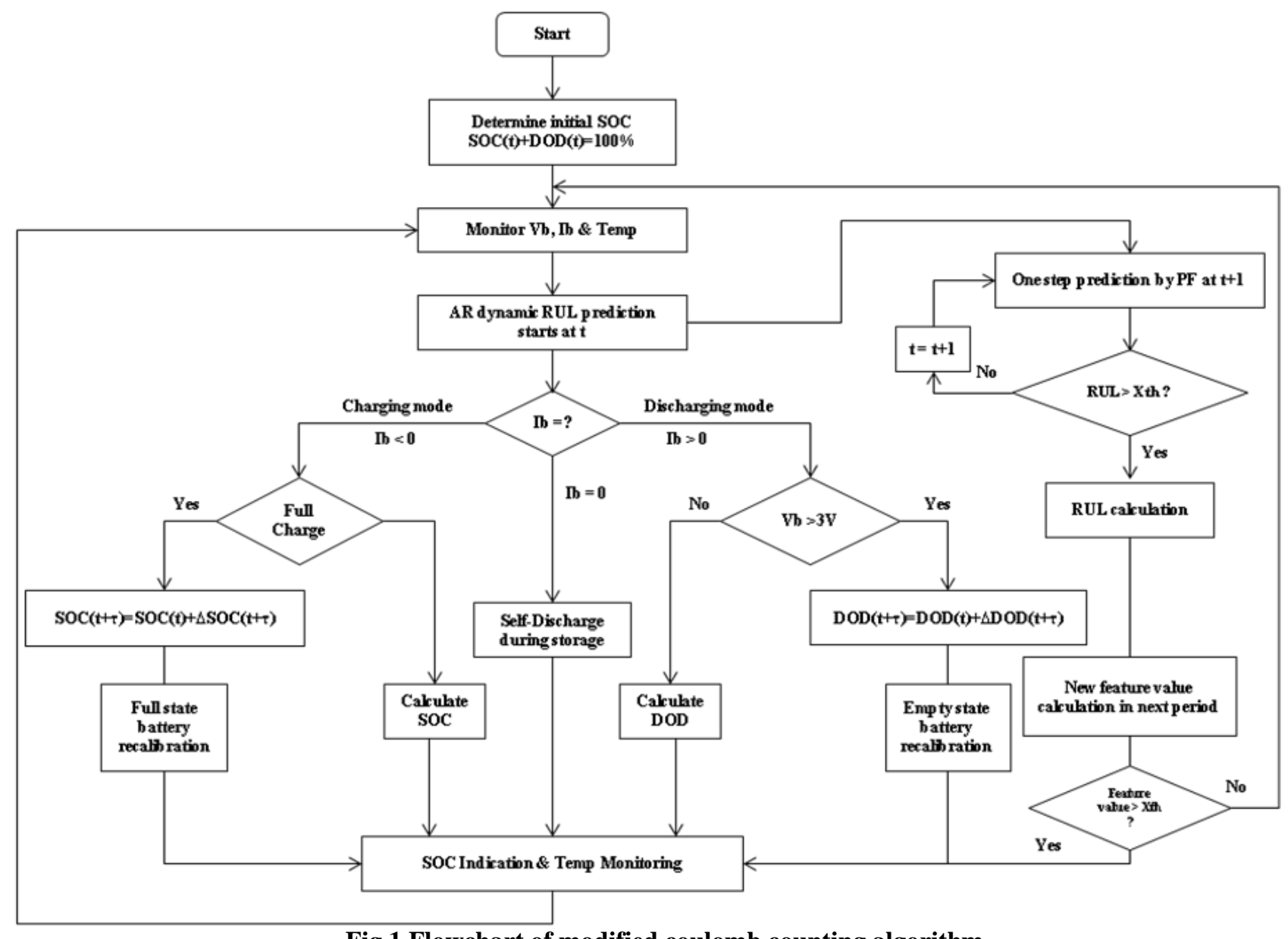

Fig.1 Flowchart of modified coulomb counting algorithm

\section{COULOMB COUNTING METHOD FOR SOC ESTIMATION}

Coulomb counting method measures the left over capacity of a battery by just collecting the charge when battery charges and discharges. This method needs long time checking and remembering, was believed to be unreasonable for actual SOC estimation yet basic in confirming the precision of evaluated results from different methods [7].

Modified coulomb counting algorithm which permit to define the initial SOC utilizing SOC-VOC curve, also considers self-discharge loss and execute and re-standardize the full stage and null stage to calculate the initial SOC and bring up to date the reference capacity with a total SOC of 100 percent.

$$
S_{\text {OC }}=\frac{Q_{\text {releasable }}}{Q_{\text {rated }}} \times 100 \%
$$

A working battery's releasable capacity $Q_{\text {releasable, is the }}$ power released while it is totally discharged [28]. The SOC is therefore well-defined as the amount of the release capacity compared to the company's rated capacity, $Q_{\text {rated }}[28]$.

$$
S_{O H}=\frac{Q_{M A X}}{Q_{\text {rated }}} \times 100 \%
$$

The greatest releasable capacity is a full charged battery, $Q_{M A X}$, which may be dissimilar from the rated capacity. Overall $Q_{M A X}$, for a freshly utilized battery is somewhat not quite the same as $Q_{\text {rated }}$ and will decrease with the time spent. It appears to be utilized to test a battery's SOH [7], [28].
Fig. 1 demonstrates the flowchart of the proposed SOC estimation algorithm. If a fully charged battery is not accessible at the beginning, the initial SOC is determined by part-wise linear mapping of the VOC-SOC curve. The estimation procedure depends on checking the battery voltage, $V b$, current $I b$ and temperature [7]. For RUL estimation procedure the autoregressive (AR) model decides the present state utilizing a few past states, and it has straightforward structure, advantageous development, and high effectiveness [20]. Through heading the working current through the battery system, the battery activity mode may be identified. If, for example, the battery is open-circuited, the current is zero, a pay for self-discharge failure will be considered [7]. The DOD includes the exhaustive charge in the discharge mode and tests the battery charging process with the aggregated charge [28]. After a rectification with the charging/discharging effectiveness, an increasingly exact estimation can be accomplished [7].

When the stacked voltage $V b$ turns out to be under $3 \mathrm{~V}$ during the discharge, the battery is depleted the battery can never again be utilized in this case and must be recharged. Simultaneously, a re-adjustment to the SOH can be made. Then again, the tried battery is completely charged if $\mathrm{Vb}$ arrives at as far as possible and Ib decreases to as far as possible [7]. Another $\mathrm{SOH}$ is acquired by collecting the whole the complete charge put into the battery [7].

Published By:

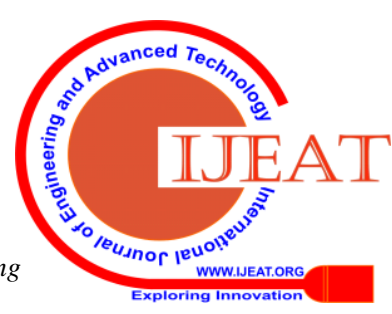




\section{A. Determination of Initial SOC}

For an EV, the most widely recognized procedure is for the initial SOC to utilize SOC-VOC work that offers the open circuit voltage to its equal SOC value. [9]. In any case, in running stage, the circumstance is extremely mind boggling. An exact SOC can't be gotten by basically aggregating the discharge current. It needs ceaseless modifications for dispensing with the errors originating from estimation float and coulombic misfortune [22]. The curve is tentatively calculated for the sake of certainty by adding a pulse charge to the Lithium-ion battery. Whereas the fact that the proposed models for the SOC-VOC function are exhaustive, modeling errors arise from fitting the experimental SOC-VOC curve equation. The nonlinearity of the system also extends the multi-faceted nature of the estimator stabilization and performance monitoring [21].

In this foreseen algorithm, we initiated by calculating the $S O C_{0}$ from determining the initial VOC, for that we are utilizing least square error curve fitting technique.

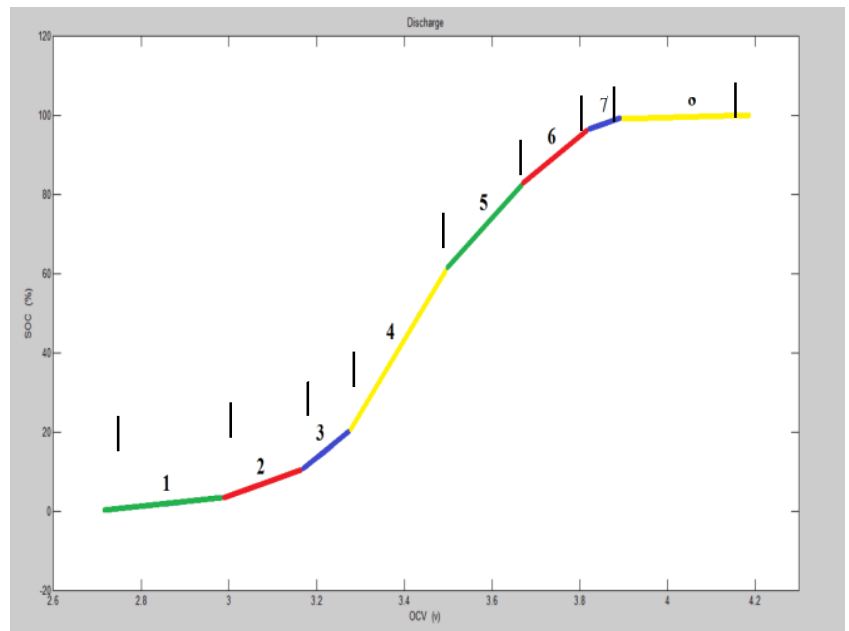

Fig.2 VOC-SOC curve part wise linear mapping

This can be approximated to a linear curve in part, thus giving the instinctive idea that it tends to be broken down into eight linear parts [23]. As appeared in Fig.2 and every piece can be stated as a linear relation as in Eq. (2):

$$
S_{O C}=f\left(V_{O C}\right)=m_{0}-V_{O C} * m_{1}
$$

We are taking variable $\mathrm{m}_{0} \& \mathrm{~m}_{1}$ differing as per VOC interims. Utilizing least square error curve fitting technique the values for $\mathrm{m}_{0}, \mathrm{~m}_{1}$ and the goodness of fit assessment factor $\mathrm{R}^{2}$, can be determined for each portion.

When the battery is charged or discharged, coulomb counter $\Delta Q$ is used for SOC tracking [9]. The quantity of charge $\Delta Q$ in a functioning period $\tau$ is acquired by temporal integration of a deliberate charging or discharging current $I_{b}$ like stated in (3):

$$
\Delta Q=\int_{t_{0}}^{t} I_{b} d t
$$

The deviation of $\Delta Q$ will be utilized In following, if equation is positive then battery is in charging and if it is negative then it is in discharging [9].
Table.1 Parameter and fitness of part wise linear segments

\begin{tabular}{|c|c|c|c|c|}
\hline Segments & VoC & $\boldsymbol{m}_{\boldsymbol{0}}$ & $\boldsymbol{m}_{\mathbf{1}}$ & $\boldsymbol{R}^{\mathbf{2}}$ \\
\hline $\mathbf{1}$ & {$[2.714 ; 3.0]$} & 13.74 & 37.62 & 0.9444 \\
\hline $\mathbf{2}$ & {$[3.0 ; 3.18]$} & 40.63 & 118.4 & 0.9820 \\
\hline $\mathbf{3}$ & {$[3.18 ; 3.24]$} & 89.25 & 272.8 & 0.9959 \\
\hline $\mathbf{4}$ & {$[3.24 ; 3.450]$} & 185.7 & 588.1 & 0.9953 \\
\hline $\mathbf{5}$ & {$[3.450 ; 3.631]$} & 137.9 & 421.5 & 0.9967 \\
\hline $\mathbf{6}$ & {$[3.631 ; 3.810]$} & 96.62 & 272.5 & 0.9993 \\
\hline $\mathbf{7}$ & {$[3.810 ; 3.946]$} & 32.12 & 26.62 & 0.9573 \\
\hline $\mathbf{8}$ & {$[3.946 ; 4.182]$} & 1.633 & -93.17 & 1.0000 \\
\hline
\end{tabular}

\section{B. RUL Estimation}

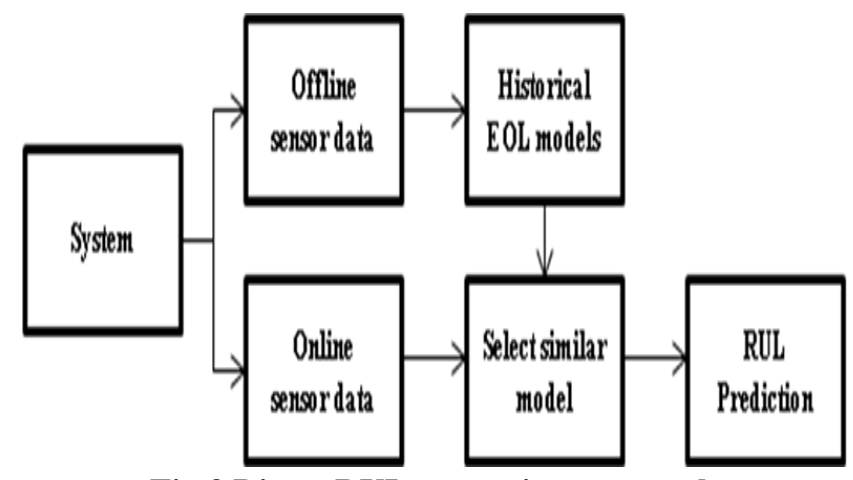

Fig.3 Direct RUL - mapping approach

For the RUL estimation aggregate debasement prognostics method has performed by modeling the damage propagation growth of the anticipated segment using sensor data. This model is utilized for health assessment and RUL. So the health pointers are take out from the raw observing signals, which may have begun from a solitary or various sensors totaled to signify the dreadful conditions development over time. The relations between such health pointers and RUL are mapped consuming diverse data-driven models. Though, the fundamental impediment of data driven methods is that it predominantly relies upon the chronicled data acquired from the critical systems over many run to failure iterations.

To overcome this data-driven model issue the enhanced particle filter is used. The enhanced particle filter for rolling bearing RUL estimation is done with help of state model and measurement model. This model is well described the system and real-time requirement. Since the bearing condition advancement relies upon past state as well as on a few $p$-step-before. After autoregressive (AR) model decides the present state utilizing a few past state, which gives high proficiency [20]. The calculation of RUL is defined by three phases in the process of safety, depletion and failure. Estimation of rolling bearing RUL is frequently performed in the stage of debasement to determine the extent to which the bearing can reach the final stage of failure. A projected bearing condition can be achieved after AR modeling and PF estimation [20]. If the anticipated status surpasses a predefined failure threshold, the estimation will be terminated and the RUL will be gained in time $t$. 


$$
R U L_{t}=t_{r}-t
$$

Where

$t$ : Present time.

$t_{r}$ :Time when the estimated condition crosses the predetermined edge of failure [20].

But the RUL we are getting is not so accurate; it has prediction error around $10 \%$.

\section{Charge Mode}

Fig. 4 shows the relation among time plus charge current throughout the constant voltage period and the relationship between time and voltage throughout the constant current stage [7].

In the CC-CV mode, the Li-ion battery charges. While a battery is in charging condition, battery voltage and current variation. In this examination, the tried battery being tested is charged primarily by a consistent rate of $0.7 \mathrm{C}$ at a threshold voltage of $4.2 \mathrm{~V}$, and subsequently by a constant voltage of $4.2 \mathrm{~V}$ at fully capacity [7]. The battery voltage rises step by step with a constant charging current and touches the threshold after $0.9 \mathrm{~h}$. Once the battery is charged by the constant-current mode, the charging current falls quickly first and gradually afterwards. Ultimately, when it is fully charged, the current declines to almost zero [28].

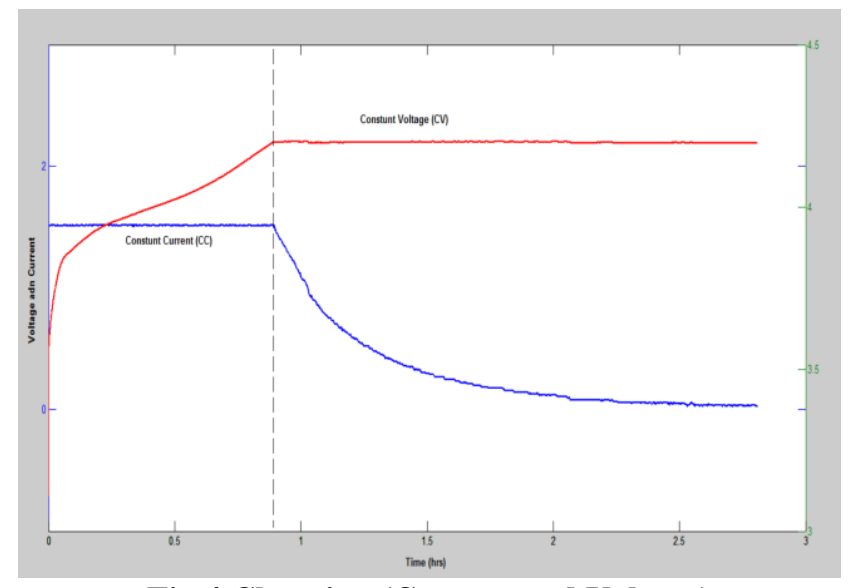

Fig.4 Charging (Current and Voltage)

For the Li-ion battery SOC calculation, the charging quantity is determined by Eq. (7) for the operating time $\tau$. And the state of charge variation is obtained by Eq. (8) for the same operating period and instantaneous value of SOC by Eq. (9).

The Coulomb counter is exhibited by gained Qobtained as stated in Eq. (7), which signifies the amount of charge collected in the course of operating period equivalent to $\tau$ [9].

$$
Q_{\text {obtained }}(t+\tau)=Q_{\text {obtained }}(t)+\Delta Q
$$

Therefore, in this equivalent operating time, the variety of the SOC has been in Eq. (7) Take a look at it. [9].

$$
\Delta S_{O C}(t+\tau)=\frac{Q_{\text {obtained }}\left(t_{0}\right)}{Q_{\text {rated }}} * 100
$$

By collecting the prior and the acquired SOC sign, we can get the immediate SOC estimate as stated in (9) [9].

$$
S_{O C}(t+\tau)=S_{O C}(t)+\Delta S_{O C}(t+\tau)
$$

The relationship shown in Eq. (7) is realized. In each charging operation, the calculation of the depth of discharge is refreshed to get it back in each switch to the discharge mode. [9].

$$
S_{O C}(t)+D_{O D}(t)=100
$$

\section{Discharge Mode}

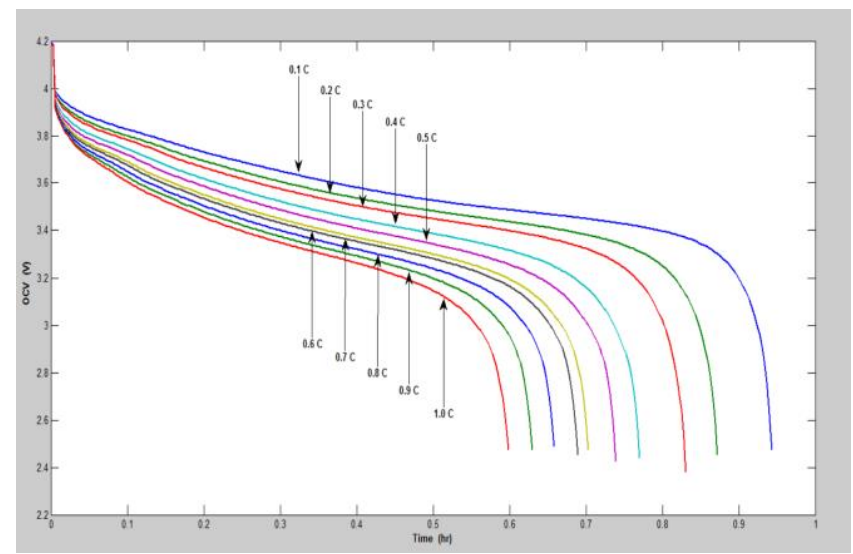

Fig.5. Discharging voltage beneath different C-rate

Fig. 5 demonstrates the curves of the discharging voltage where various Current-rate discharges from a Li-ion battery. The voltage of the terminal decays as the functioning time slips away [7]. A greater current allows the terminal voltage to drop more quickly, prominent to a shortened functioning time [28]. As indicated in Fig. 5, if battery discharged by 0.1 $\mathrm{C}$, then the battery will continue for $58 \mathrm{~min}$, however only 37 min for a high discharge level of $1 \mathrm{C}$. Clearly minor differences, however, are $1.8 \%$ of the rated capacity [7].

At the point when a battery is discharged, the DOD may be specified as the percentage of the discharged capacity for $Q_{\text {rated }}[28]$.

$$
D_{O D}=\frac{Q_{\text {lost }}}{Q_{\text {rated }}} \times 100 \%
$$

The quantity of charge is lost during the functioning period $\tau$ that signifies by the $Q_{\text {lost }}$ which represents coulomb counter [9].

$$
Q_{\text {lost }}(t+\tau)=Q_{\text {lost }}(t)+\Delta Q
$$

The deviation of depth of discharge is determined by (13) and (14) to compute value of DOD [9].

$$
\Delta D_{O D}(t+\tau)=\frac{Q_{\text {lost }}\left(t_{0}\right)}{Q_{\text {rated }}} * 100
$$

Where

$$
D_{O D}(t+\tau)=D_{O D}(t)+\Delta D_{O D}(t+\tau)
$$

For the estimation of Li-ion battery, the amount of charge losses is calculated by Eq. (12) for operating period $\tau$. And the depth of discharge variation is obtained by Eq. (13) for the same operating period and instantaneous value of DOD by Eq. (14). 


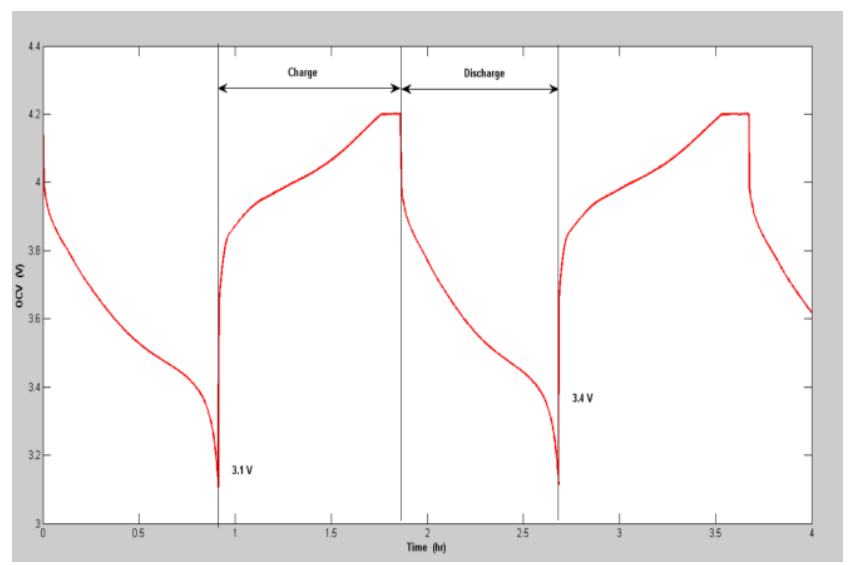

Fig.6. Battery's discharge curves

The Li-ion battery discharge curve for the dissimilar DOD is displayed in Fig. 6. After some time interval, the battery is charged and discharged (randomly) to determine the maximum capacity of the Li-ion battery. The battery is fully charged till $4.2 \mathrm{~V}$ when discharged at various cutoff voltages. Consequently, test is there to evaluation of extreme capacity with a variety of current loads and various depth of discharge [27].

\section{E. Self-Discharge}

The Li-ion battery self-discharge is shared phenomena, which affect SOC estimation. Self-discharge is stated as a charge loss with storing time, which relies upon several elements comprising of ambient temperature, cycle times and storing time considering that batteries reaches $6 \%$ of self-discharge rate per month [27], the quantity of charge losses per 60 minutes is deliberated. This quantity is calculated by constant, $q_{\text {per/hour }}$ [9]. At that time, the amount of charge degenerate to this stage $Q_{o c}$ is determined by Eq. (15), demonstrating the collective losses throughout the storing time [9].

$$
Q_{o c}(h+1)=Q_{o c}(h)+q_{\text {per } / \text { hour }}
$$

Where,

\section{$h$ : Storing time}

Finally the obtained value will be include to the quantity of charge lost $Q_{\text {lost }}$ all through the discharge mode and withdrawn from the quantity of charge stored $Q_{\text {gained }}$ in the charging mode as stated in Eq. (16) and (17) [9]:

$$
\begin{aligned}
Q_{\text {lost }} & =Q_{\text {lost }}+Q_{o c} \\
Q_{\text {tot }} & =Q_{\text {tot }}-Q_{o c}
\end{aligned}
$$

\section{F. Temperature Effect}

Thermal characteristics \& tendencies of Li-ion batteries are assumed by battery temperature supervision. The impact of temperature deviation with capacity of a battery shows on SOC estimation. The ambient temperature is comparative to the capacity of the battery [24]. In fig. 7 at short rate the released capacity from the battery declines radically one can see that the capacity is around $800 \mathrm{mAh}$ when the thermal energy is about $0^{\circ} \mathrm{C}$ and touches to $580 \mathrm{mAh}$ when temperature is $-20^{\circ} \mathrm{C}$.

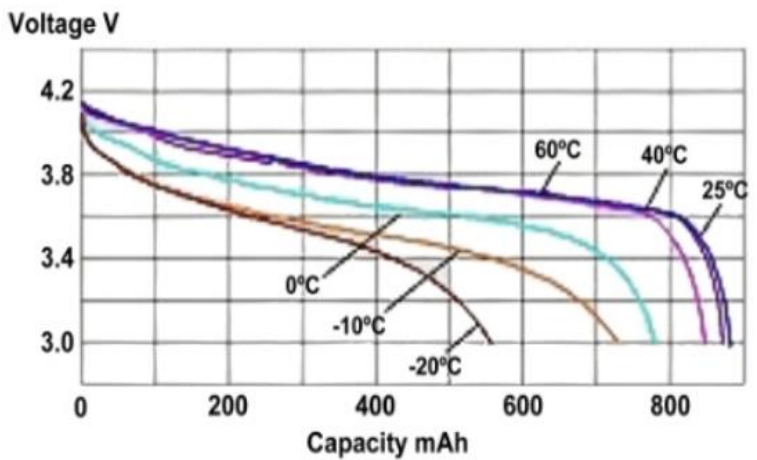

Fig.7 A typical discharge profile when battery is under various temperatures [25].

The temperature impact is also noticed on VOC-SOC model particularly in the VOC range in the middle of $3-3.3 \mathrm{~V}$ as displayed in fig.8.
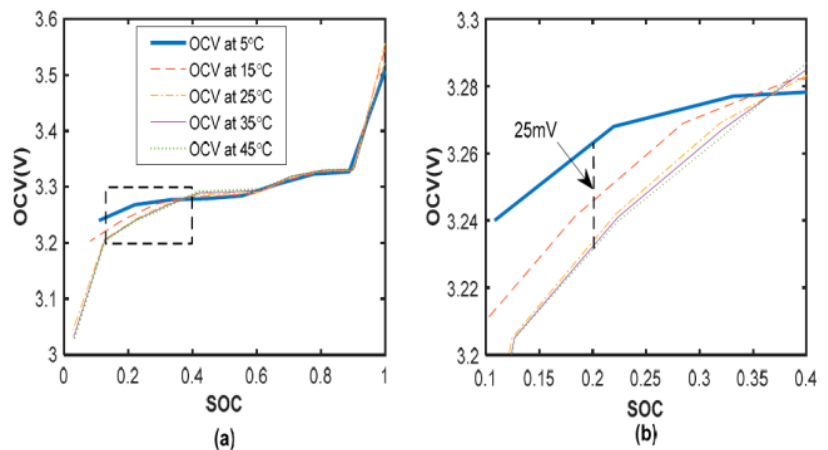

Fig.8. VOC-SOC variations under various temperatures [26]

The VOC was resolved from the Pulse Discharge Experiment. The VOC is the battery's terminal voltage at balance stage got throughout the moderation time. As observed in Fig.7, a VOC-SOC curve at a variety of temperatures is plotted. In Fig. 8a, it is seen that VOC is constant for the SOC value from range of 0.1 to 0.9 . As observed in Fig. 8b, a dissimilar VOC at different temperatures can be detected. For 0.2 SOC value, the VOC is more or less $25 \mathrm{mV}$ with a fault of $10 \%$ in State of Charge [26]. Concerning the fig.7, so as to consider the ambient temperature variation, we suggest utilizing a different constant $\alpha$ to load the SOC value such to adjust it to the correspondent external temperature.

Table.2 Variant Co-efficient $\boldsymbol{\alpha}$ at different temperatures

\begin{tabular}{|c|c|c|c|c|c|}
\hline Temperature & $-10^{\circ} \mathrm{C}$ & $5^{\circ} \mathrm{C}$ & $15^{\circ} \mathrm{C}$ & $25^{\circ} \mathrm{C}$ & $45^{\circ} \mathrm{C}$ \\
\hline$\alpha$ & 0.5 & 0.6 & 0.8 & 1 & 0.9 \\
\hline
\end{tabular}

For precision of SOC estimation we reduced the variant coefficient $\alpha$ and an improved SOC estimation method has been proposed, which is shown in formula (18).

$$
S_{O C}(t)=S O C_{0}+\frac{1}{C_{\text {nom }}} \int_{t_{0}}^{t} I_{b} d t-\alpha
$$




\section{HARDWARE IMPLEMENTATION}

The proposed modified coulomb counting algorithm with RUL estimation has imposed on hardware base. Where to monitor the measured key parameters of a battery such as voltage $V b$, current $I b$ and Temperature has taken into account and these parameters are read by the 10 bits to ADC microcontroller unit PIC18F.

\section{A. Measurement}

Generally parameters of the battery recognition are the most difficult problem in BMS for control and management. The BMS is comprised of cell voltage reader, current transducer and battery health monitor. To estimate state of charge, all available batteries require cell voltage accuracy. This cell voltage may vary by the health of current condition of battery. Fig. 9 shows different types of module are connected in data gaining system.

In fig.9 a basic circuit is developed where a sensing resistor $R_{\text {sens }}$ is positioned to detect charge as well as discharge voltages and current of the Li-ion battery.

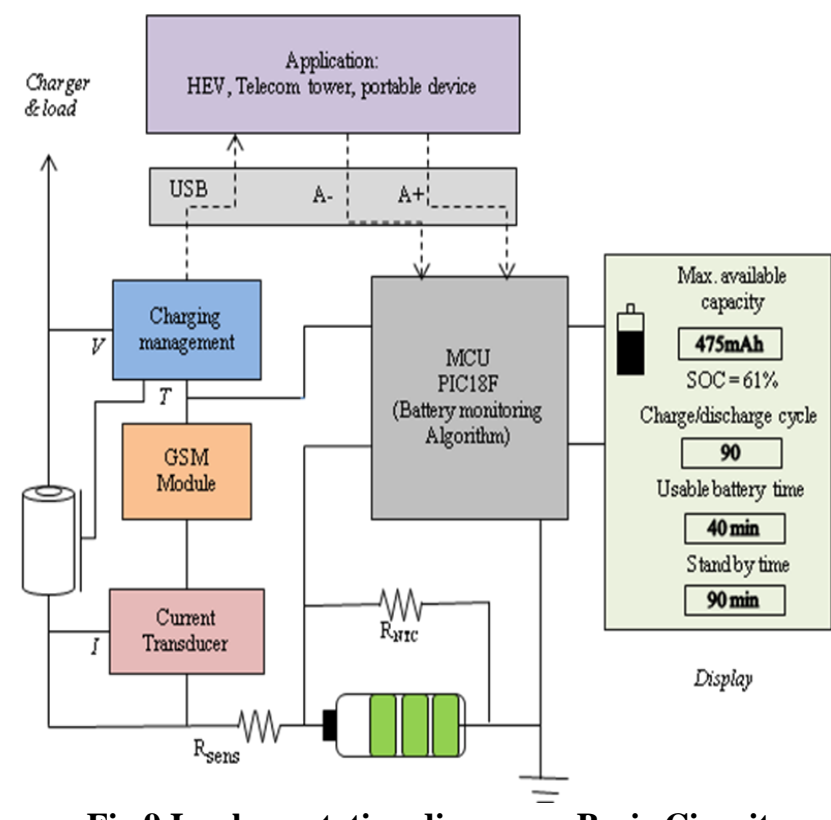

Fig.9 Implementation diagram - Basic Circuit

The voltage $V b$ can be identified by reading voltage across battery circuit, and current can be identified by reading voltages at two terminals of resistor (along with noted resistance values). Current transducer HO-P series is used for measurement of AC current data. It is an open loop multi-range current transducer having single power supply $+5 \mathrm{~V}$. The current transducers over current detection peak value is about $2.93 * I_{P N}$ and low power consumption.

Resistance to be make sure, during charging at constant 1C-rate (current rate) not to be applied high that would cause power dissipation but fair enough to allow ADC microcontroller to detect the voltage variations (probably $\mathrm{I}=10 \mathrm{~mA}$ ) and identify the charging end point as well.

For instance, 0.2 Ohms resistance should be accurate to detect $12 \mathrm{~mA}$ current (for $U=1 \mathrm{mV}$ ) in order not to dissipate much energy when charging with maximum current value $(\mathrm{P}=100 \mathrm{~mW})$. Our goal is to detect voltage difference of $1 \mathrm{~mA}$ using 10 bit converter.

To identify remaining useful life (RUL), rolling bearing is used along with particle filter PF. To monitoring the SOC status of a battery with IoT we have used SIM800L is a portable GSM breakout board with all the capabilities of the larger SIM900 shields, which sends data to the web server using GPRS. The board draws a highest of 2A current in between voltage input of $3.7 \mathrm{~V}$ to $4.2 \mathrm{~V}$ as shown in Fig.10.

Between MCU and Application, USB connection is placed to acquire battery supplied parameter readings.

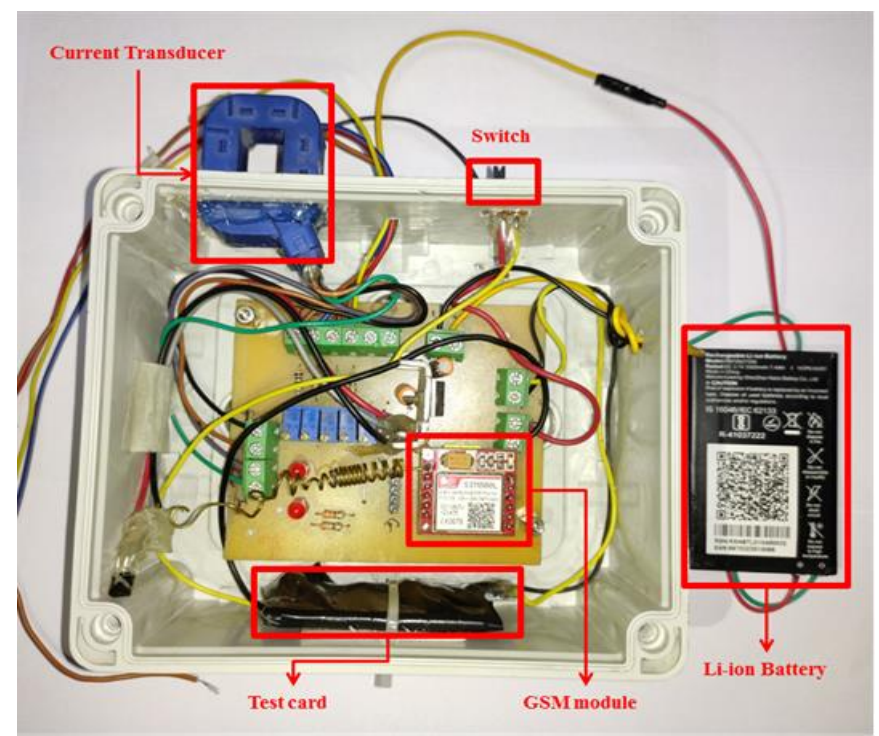

Fig.10 Implementation of actual coulomb-counting

To detect the ambient temperature, $R_{N T C}$ (Negative temperature coefficient resistor) is placed. This MCU's (PIC18F) thermistor biased by the IO and closely connected to batteries as described under fig.9.

Fig.10. shows the actual implementation of proposed modified coulomb counting algorithm.

\section{B. Experimented Results}

After completing the integration part of the algorithm, we did some testing on an Electric Vehicle battery to validate our algorithm performance. Next, we experimented with data simulated on MATLAB for coulomb counting with data obtained from the MCU where modified algorithms were implemented to prove accuracy. This whole experiment took on a $4.2 \mathrm{~V}$ li-ion battery discharge data.

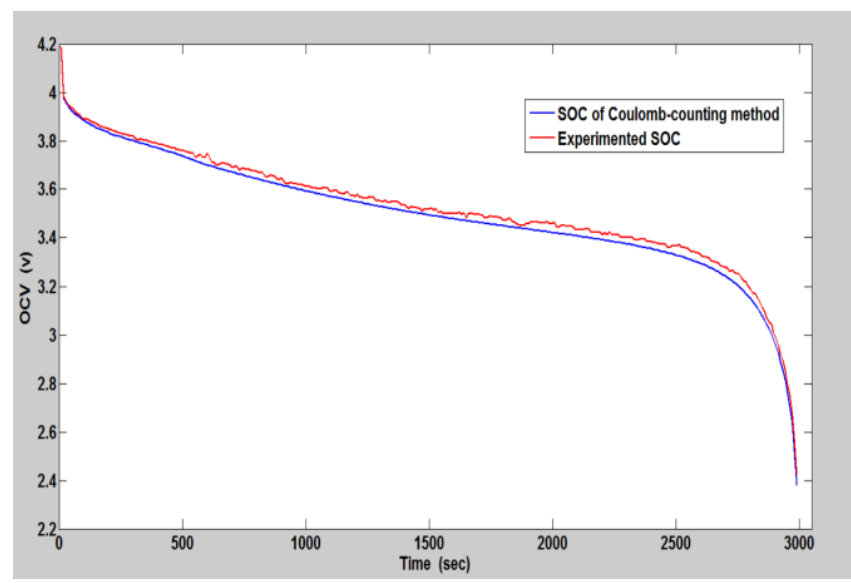

Fig.11 Instantaneous values for SOC of coulomb counting and experimented SOC 
The blue curve displays the simulated coulomb counting SOC on MATLAB in Fig. 11, and the red colored curve shows the Experimented SOC obtained from the MCU (PIC18F) embedded algorithm. The variation exists among two curves and the explanation behind that there are certain parameters that cause inaccuracy if we seek to estimate the State of Charge of the battery.

\begin{tabular}{l} 
Table.3 Simulated SOC and PIC18F obtained SOC \\
\begin{tabular}{|c|c|c|}
\hline $\begin{array}{c}\text { Open Circuit } \\
\text { Voltage }\end{array}$ & $\begin{array}{c}\text { State of } \\
\text { Charge of } \\
\text { CC }\end{array}$ & $\begin{array}{c}\text { Experiment } \\
\text { ed SOC }\end{array}$ \\
\hline 4.18 & $100 \%$ & $100 \%$ \\
\hline 3.75 & $85 \%$ & $83.17 \%$ \\
\hline 3.65 & $75 \%$ & $72.07 \%$ \\
\hline 3.56 & $62 \%$ & $59.08 \%$ \\
\hline 3.49 & $50 \%$ & $46.09 \%$ \\
\hline 3.42 & $34 \%$ & $31.40 \%$ \\
\hline 3.38 & $25 \%$ & $21.78 \%$ \\
\hline 3.26 & $10 \%$ & $8.37 \%$ \\
\hline 2.91 & $0 \%$ & $0 \%$ \\
\hline
\end{tabular} \\
\hline
\end{tabular}

$$
S_{O C}(t)=S O C+(\alpha+\beta+\delta)
$$

This $\alpha, \beta, \delta$ parameters are considered as temperature, load current and duty cycle this causes inaccuracy. After the data is sent to IoT and at the time of data-analysis if we incorporate these parameters we can get more accurate results.

\section{CONCLUSION}

So throughout the experiment, we have explored the coulomb counting algorithm implemented over Lithium-ion battery. We understood the SOC workflow through algorithm. On initial estimation of SOC we used VOC-SOC function to generate linear approximation mapping which gives it as eight piece separated readings. This has helped us to identify the battery's actual SOC estimation using derived formulas and applied circuit parameters (Current, Voltage).

We have explored RUL estimations in Lithium-ion batteries. With variant temperatures and applied parameters, we studied the behavior patterns of battery charging and discharging abilities.

Also, we have tested MCU (PIC18F) hardware along with IoT device which can sends data to web server using GPRS. On few hardware consistency tests, the error estimation of the instantaneous capacities has found to be only about $3 \%$ is pretty adequate. With all observations and followed steps, we may conclude that, the proposed Modified Coulomb-counting method can be concluded as implementable in electric vehicles, portable devices and other various devices.

\section{REFERENCES}

1. T. H. Wu, J. K. Wang, C. S. Moo, and A. Kawamura, "State-of-charge and state-of-health estimating method for lithium-ion batteries," in 2016 IEEE 17th Workshop on Control and Modeling for Power Electronics (COMPEL), pp. 1-6, June 2016.

2. Y. Nishi, "Lithium ion secondary batteries; past 10 years and the future," Journal of Power Sources, vol. 100, no. 1, pp. 101-106, 2001.

3. Plett, G.L. Extended Kalman filtering for battery management systems of LiPB-based HEV battery packs:Part 1. Background. J. Power Sources 2004, 134, 252-261.

4. Bhangu, B.S.; Bentley, P.; Stone, D.A.; Bingham, C.M. Nonlinear observers for predicting state-of-charge and state-of-health of lead-acid batteries for hybrid-electric vehicles. IEEE Trans. Veh. Technol. 2005, 54, 783-794.

5. W.-Y. Chang, "The state of charge estimating methods for battery: a review," ISRN Applied Mathematics, vol. 2013, 2013.

6. S. Piller, M. Perrin, and A. Jossen, "Methods for state-of charge determination and their applications," Journal of power sources, vol. 96, no. 1, pp. 113-120, 2001.

7. Kong. Soon. Ng, Chin-Sien Moo, Yi-Ping Chen, and Yao-Ching Hsieh, "Enhanced coulomb counting method for estimating state-of-charge and state-of-health of lithium-ion batteries," Applied energy, 2009.

8. I. Baccouche, A. Mlayah, S. Jemmali, B. Manai, and N. Essoukri Ben Amara, "Implementation of a coulomb counting algorithm for soc estimation of li-ion battery for multimedia applications," in Systems, Signals \& Devices (SSD), 12th International Multi-Conference on, pp. 1-6, IEEE, 2015.

9. I. Baccouche, A. Mlayah, S. Jemmali, B. Manai, and N. Essoukri Ben Amara, "Implementation of an Improved Coulomb-Counting Algorithm Based on a Piecewise SOC-VOC Relationship for SOC Estimation of Li-Ion Battery," International journal of renewable energy research, pp. 11-6, IEEE, 2017

10. Z. Zou, J. Xu, C. Mi, B. Cao, and Z. Chen, "Evaluation of model based state of charge estimation methods for lithium-ion batteries," Energies, vol. 7, no. 8, pp. 5065-5082, 2014.

11. P. Singh, C. Fennie, and D. Reisner, "Fuzzy logic modelling of state-of-charge and available capacity of nickel/metal hydride batteries," Journal of Power Sources, vol. 136, no. 2, pp. 322-333, 2004.

12. R. Li, J. F. Wu,H. Y.Wang, and G. C. Li, "Prediction of state of charge of lithium-ion rechargeable battery with electrochemical impedance spectroscopy theory," in Proceedings of the 5th IEEE Conference on Industrial Electronics and Applications (ICIEA'10), pp. 684-688, Taichung, Taiwan, June 2010.

13. H. He, R. Xiong, and J. Fan, "Evaluation of lithium-ion battery equivalent circuit models for state of charge estimation by an experimental approach," Energies, vol. 4, no. 4, pp. 582-598, 2011.

14. G. L. Plett, "Extended kalman filtering for battery management systems of lipb-based hev battery packs: Part 2. modeling and identification," Journal of power sources, vol. 134, no. 2, pp. 262-276, 2004.

15. F. Huet, "A review of impedance measurements for determination of the state-of-charge or state-of-health of secondary batteries," Journal of Power Sources, vol. 70, no. 1, pp. 59-69, 1998.

16. S. Abu-Sharkh and D. Doerffel, "Rapid test and non-linear model characterisation of solid-state lithium-ion batteries," Journal of Power Sources, vol. 130, no. 1-2, pp. 266-274, 2004.

17. S. Sato and A. Kawamura, "A new estimation method of state of charge using terminal voltage and internal resistance for lead acid battery," in Proceedings of the Power Conversion Conference, pp. 565-570, Osaka, Japan, April 2002.

18. Y. Shen, "Adaptive online state-of-charge determination based on neuro-controller and neural network," Energy Conversion and Management, vol. 51, no. 5, pp. 1093-1098, 2010.

19. P. Singh, C. Fennie, and D. Reisner, "Fuzzy logic modelling of state-of-charge and available capacity of nickel/metal hydride batteries," Journal of Power Sources, vol. 136, no. 2, pp. 322-333, 2004.

20. Y. Qian and R. Yan, "Remaining Useful Life Prediction of Rolling Bearings Using an Enhanced Particle Filter," Senior member, ieee transactions on instrumentation and measurement, 2015.

21. H. Rahimi-Eichi, F. Baronti, and M. Y. Chow, "Modeling and online parameter identification of Li-Polymer battery cells for SOC estimation," in Proc. IEEE ISIE, 2012, pp. 1336-1341. 
22. Li Zhao, Muyi Lin and Yong chen, "Least-squares based coulomb counting method and its application for state-of-charge (SOC) estimation in electric vehicles," international journal of energy research 2016.

23. I. Baccouche, S. Jemmali, B. Manai, R. Chaibi, and N. E. B. Amara, "Hardware implementation of an algorithm based on kalman filtrer for monitoring low capacity li-ion batteries,", 7th International Renewable Energy Congress (IREC), pp. 1-6, March 2016.3):

24. Zhang SS, Xu K, Jow TR, "Electrochemical impedance study on the low temperature of Li-ion batteries," Electrochim Acta 2004;49:1057-61.

25. http://www.ibt-power.com/Battery_packs/Li_Polyme/Lithium_polymer tech.html

26. Cheng Siong Chin and Zuchang Gao , "State-of-Charge Estimation of Battery Pack under Varying Ambient Temperature Using an Adaptive Sequential Extreme Learning Machine,” Energies 2018, 11, 711.

27. M. A. Hannan, M.S.H. Lipu, A. Hussain, A. Mohamed, "A review of lithium-ion battery state of charge estimation and management system in electric vehicle applications: Challenges and recommendations," Renewable and Sustainable Energy, 2017.

28. M. Murnane, A. Ghazel, "A Closer Look at State of Charge (SOC) and State of Health (SOH) Estimation Techniques for Batteries," in technical article, analog devices.

\section{AUTHORS PROFILE}

Umang Dongre, B. E. graduate majored in Electronics and Telecommunication Engineering from Amravati University in year 2016 pursuing M.Tech in VLSI Design from Shri Ramdeobaba College of Engineering and Management, Nagpur University. 